\title{
Species convergence into life-forms in a hyperseasonal cerrado in central Brazil
}

\author{
Silva, IA. * and Batalha, MA. \\ Laboratório de Ecologia Vegetal, Departamento de Botânica, Universidade Federal de São Carlos - UFSCar, \\ CP 676, CEP 13565-905, São Carlos, SP, Brazil \\ *e-mail: igor6cordas@yahoo.com.br
}

Received September 21, 2006 - Accepted November 30, 2006 - Distributed May 31, 2008

(With 3 figures)

\begin{abstract}
Whether the functional structure of ecological communities is deterministic or historically contingent is still quite controversial. However, recent experimental tests did not find effects of species composition variation on trait convergence and therefore the environmental constraints should play the major role on community convergence into functional groups. Seasonal cerrados are characterized by a sharp seasonality, in which the water shortage defines the community functioning. Hyperseasonal cerrados experience additionally waterlogging in the rainy season. Here, we asked whether waterlogging modifies species convergences into life-forms in a hyperseasonal cerrado. We studied a hyperseasonal cerrado, comparing it with a nearby seasonal cerrado, never waterlogged, in Emas National Park, central Brazil. In each area, we sampled all vascular plants by placing 40 plots of $1 \mathrm{~m}^{2}$ plots in four surveys. We analyzed the species convergences into life-forms in both cerrados using the Raunkiaer's life-form spectrum and the index of divergence from species to life-form diversity (IDD). The overall life-form spectra and IDDs were not different, indicating that waterlogging did not affect the composition of functional groups in the hyperseasonal cerrado. However, there was a seasonal variation in IDD values only in the hyperseasonal cerrado. As long as we did not find a seasonal variation in life-form diversity, the seasonal variation of convergence into life-forms in the hyperseasonal cerrado was a consequence of the seasonal variation of species diversity. Because of high functional redundancy of cerrado plants, waterlogging promoted a floristic replacement without major changes in functional groups. Thus, waterlogging in the hyperseasonal cerrado promoted seasonal changes in species convergence into life-forms by reducing species diversity.
\end{abstract}

Keywords: hyperseasonal cerrado, IDD, life-form, species diversity, trait convergence.

\section{Convergência de espécies em formas de vida em um cerrado hiperestacional no Brasil central}

\section{Resumo}

Se a estrutura ecológica das comunidades é determinística ou historicamente dependente é ainda um tema controverso. Entretanto, testes experimentais recentes não encontraram efeitos da variação da composição de espécies na convergência de traços funcionais e, portanto, as restrições ambientais devem ter um papel principal na convergência das comunidades em grupos funcionais. Cerrados estacionais são caracterizados por uma estacionalidade pronunciada, em que a seca define o funcionamento da comunidade. Cerrados hiperestacionais experimentam adicionalmente um alagamento na estação chuvosa. Aqui, perguntamo-nos se o alagamento modifica a convergência de espécies em formas de vida em um cerrado hiperestacional. Para tanto, estudamos um cerrado hiperestacional, comparando-o com um cerrado estacional, nunca alagado, no Parque Nacional das Emas, GO. Em cada cerrado, usamos 40 parcelas de $1 \mathrm{~m}^{2}$ e amostramos todas as plantas vasculares. Analisamos a convergência de espécies em formas de vida usando o espectro biológico de Raunkiaer e o índice de divergência entre a diversidade de espécies e de formas de vida (IDD). Os espectros gerais e os IDDs não diferiram, indicando que o alagamento não afetou a composição dos grupos funcionais no cerrado hiperestacional. Entretanto, houve uma variação estacional nos valores de IDD somente no cerrado hiperestacional. Como não observamos variações estacionais na diversidade de formas de vida, a variação da convergência no cerrado hiperestacional foi uma consequiência da variação estacional da diversidade de espécies. Devido à elevada redundância funcional das plantas do cerrado, o alagamento promoveu substituições florísticas sem maiores mudanças nos grupos funcionais. Portanto, o alagamento promoveu mudanças estacionais na convergência de espécies em formas de vida, reduzindo a diversidade de espécies.

Palavras-chave: cerrado hiperestacional, convergência funcional, diversidade de espécies, formas de vida, IDD. 


\section{Introduction}

Whether the functional group structure of ecological communities is deterministic or historically contingent is still quite controversial (Chase, 2003). The deterministic view suggests that communities converge towards a common structure determined by environmental conditions, irrespective of the history of community assembly (Fukami et al., 2005). The alternative view suggests that stochastic forces producing variation in the sequence and timing of species arrivals can cause divergence in community structure among localities, even under identical environmental conditions and regional species pool (Fukami et al., 2005). A reconcilable view, however, suggests that whether communities converge or diverge depends on the level of community organization considered. According to this, community assembly is deterministic in the general composition of functional groups, but historically contingent in the composition of species (Temperton et al., 2004; Fukami et al., 2005). Despite the possible relative influence of environment and history on assemblage composition (Gentry, 1988; Kinzig et al., 1999; Chase, 2003), recent experimental tests did not find effects of species composition variation on trait-group composition (Fukami et al., 2005). As a result, environmental constraints would play the major role on community convergence into functional groups (Fukami et al., 2005).

Ricotta et al. (2002) proposed an index of divergence from species to functional group diversity that is useful to measure species convergence of a given community into ecologically relevant groups irrespective of their taxonomic classification. The index of intrinsic diversity divergence (IDD) is based on the notion of intrinsic diversity ordering in which community $\mathrm{A}$ is intrinsically more diverse than community $\mathrm{B}$ if and only if community $\mathrm{A}$ has its diversity profile everywhere above that of community B (for details see Patil and Tailie, 1979; 1982). The IDD is obtained by comparing the area under the species diversity profile with the area under the corresponding functional group diversity profile (Ricotta et al., 2002). The IDD may range from zero to one and, when one uses species life-form, IDD is also a structural measure of communities (Ricotta et al., 2002). Consequently, communities that are structurally more homogeneous should present higher IDD values than less homogeneous communities (Ricotta et al., 2002).

Raunkiaer (1934) proposed a system to classify plant life-forms based on the position and protection degree of the regeneration buds. In his classification, there are five major classes, arranged according to increased protection of the regeneration buds: phanerophytes, chamaephytes, hemicryptophytes, cryptophytes, and therophytes. Accordingly, harsh growing conditions should favour species with periodic reduction to a remnant shoot system that either lies flat on the ground (hemicryptophytes) or is embedded in the soil (cryptophytes) (Cornelissen et al., 2003). Therophytes are expected to prevail where growing conditions become so extreme that the probability of survival to the second year becomes very small (Bazzaz and Morse, 1991). Although sometimes strongly criticized (e.g., Sarmiento and Monasterio, 1983), Raunkiaer's system is the simplest and, in many ways, the most satisfying classification of plants into life-forms (Begon et al., 1996). His system has gained wide acceptance in the sense that publications report life-form categories as a matter of routine (Westoby, 1998) and, thus, is suitable for the scope of our study.

Seasonal savannas are characterized by a sharp seasonality, in which the pronounced rainless season defines the community functioning (Sarmiento, 1983). Phanerophytes, chamaephytes, and hemicryptophytes are generally well represented in life-form spectra of Brazilian seasonal savannas (see Batalha and Martins, 2002 for references). Hyperseasonal savannas are characterized by the alternation of two contrasting stresses during each annual cycle, one induced by drought; the other, by waterlogging in some months in the rainy season (Sarmiento et al., 2004). The consequences of waterlogging to savanna species can be fatal, because, as aerobic respiration ceases, levels of energy-rich adenylates drop rapidly, causing a dramatic decline in ion uptake and transport (Crawford, 1982; Koslowski, 1997). It is known that waterlogging determines the species abundance in hyperseasonal savannas (Sarmiento, 1983; Sarmiento, 1996; Batalha et al., 2005; Cianciaruso et al., 2005), but does it alter species convergences towards life-forms with more protected regeneration buds?

We related the first occurrence of a hyperseasonal cerrado in Emas National Park (ENP), central Brazil, flooded by the accumulation of rain water, with fewer species and less diverse than the seasonal cerrado (Batalha et al., 2005; Cianciaruso et al., 2005). We expected that the life-form spectra and IDDs could highlight the effects of waterlogging upon the convergence of cerrado species into life-forms, considering that both hyperseasonal and seasonal cerrados have the same physiognomy (Batalha et al., 2005), are on similar soils (Amorin and Batalha, 2006), and under the same climate and fire regimes (Ramos-Neto and Pivello, 2000). Our aim was to study the life-form spectrum and IDD of the hyperseasonal cerrado, comparing it to a nearby seasonal cerrado. We addressed the following questions: What are the prevailing life-forms in the hyperseasonal cerrado? Is the life-form spectrum of the hyperseasonal cerrado different from the life-form spectrum of the seasonal cerrado? Is the IDD of the hyperseasonal cerrado different from the IDD of the seasonal cerrado, that is, is the convergence of species into life-forms different between the two vegetation forms? Do the IDDs of both cerrados vary throughout the year, accompanying the climatic seasonality and environmental constraints?

\section{Material and Methods}

We carried out this study in Emas National Park (ENP), one of the largest and most important reserves 
in the cerrado region. It comprises 132,941 ha and is located in the Brazilian Central Plateau, southwestern Goiás State $\left(17^{\circ} 49^{\prime}-18^{\circ} 28^{\prime} \mathrm{S}\right.$ and $\left.52^{\circ} 39^{\prime}-53^{\circ} 10^{\prime} \mathrm{W}\right)$. We sampled the hyperseasonal cerrado (approximately, $18^{\circ} 18^{\prime} 07^{\prime \prime} \mathrm{S}$ and $52^{\circ} 57^{\prime} 56^{\prime \prime} \mathrm{W}$ ) and a nearby seasonal cerrado (approximately, $18^{\circ} 17^{\prime} 34^{\prime \prime} \mathrm{S}$ and $52^{\circ} 58^{\prime} 12^{\prime \prime} \mathrm{W}$ ) in four surveys during 2003. In each vegetation form, we placed randomly ten plots $\left(1 \mathrm{~m}^{2}\right)$ in each one of the four surveys carried out: February 2003, in mid rainy season, when the hyperseasonal cerrado was waterlogged; May 2003, in late rainy season; August 2003, in the dry season; and November 2003, in early rainy season. For a detailed description of the studied areas and sampling methods, see Batalha et al. (2005), Cianciaruso et al. (2005), and Amorin and Batalha (2006).

We assigned the life-form of each species with an identification key (Mueller-Dombois and Ellenberg, 1974), considering only Raunkiaer's major classes - phanerophytes, chamaephytes, hemicryptophytes, cryptophytes, and therophytes - and including lianas in the "phanerophyte" class and geophytes in the "cryptophyte" class, as originally proposed by Raunkiaer (1934). We used data from all surveys to construct the life-form spectra and to compute the overall IDDs of cerrados $(n=40)$. For seasonal comparisons of IDDs, we used the data from each survey $(n=10)$. To check whether the life-form spectrum of the hyperseasonal cerrado was significantly different from the spectrum of the seasonal cerrado, we applied a heterogeneity chi-square analysis (Zar 1999). With this analysis, we determined whether the two life-form spectra came from the same data population (Zar 1999).

To determine the IDD of each cerrado, first we computed the intrinsic diversity profiles for species $\left(\alpha_{\mathrm{SP}}\right)$ and the intrinsic diversity profiles for life-forms $\left(\alpha_{\mathrm{LF}}\right)$, which are related, respectively, to the area under the species diversity profile and the life-form diversity profile. We constructed the diversity profiles following the procedures of Patil and Tailie (1979): we arranged the relative abundances in descending order and rearranged them into a cumulative relative pattern; then, we obtained the diversity profiles by joining the points given by the $\mathrm{cu}-$ mulative relative species and life-form abundances and by the cumulative species number, being $(0 ; 0)$ the first plotted points. We determined the area under a given profile as:

$$
\alpha=\mathrm{I} \times(\mathrm{N} / 2)
$$

in which:

$\mathrm{I}=\left[\left(2 \Sigma \mathrm{i} \times \mathrm{p}_{\mathrm{i}}\right)-1\right] / \mathrm{N}$ and $\mathrm{i}(1 \leq \mathrm{i} \leq \mathrm{N})$ was the rank of the relative abundances $\mathrm{p}_{\mathrm{i}}$ arranged in descending order (Ricotta et al., 2002). We calculated the index of intrinsic divergence (IDD) of each cerrado as:

$$
\mathrm{IDD}=1-\left[\left(\alpha_{\mathrm{LF}}-0.5\right) /\left(\alpha_{\mathrm{SP}}-0.5\right)\right]
$$

We applied a randomization test (Manly, 1997), with 5,000 permutations, to check whether the IDDs of the hy- perseasonal and seasonal cerrados were significantly different $(\alpha=0.05)$, using a computer routine we wrote in $\mathrm{C}^{++}$. The randomization test was applied as follows: first, we calculated the difference $\mathrm{D}_{1}$ between the IDDs of both cerrados $\left(\mathrm{D}_{1}=\left|\mathrm{IDD}_{\text {seasonal cerrado }}-\mathrm{IDD}_{\text {hyperseasonal cerrado }}\right|\right)$; then, we randomly resampled the species of both cerrados and determined the difference D between the IDDs thus obtained - a step repeated 5,000 times, providing us with a distribution of $\mathrm{D}$ values; finally, we calculated the proportion of all $\mathrm{D}$ values that were higher than or equal to $\mathrm{D}_{1}$. We used the same procedure to test for differences in the IDDs among the seasons in each vegetation form.

\section{Results}

In the hyperseasonal cerrado, we sampled 63 species (Table 1), of which nine were phanerophytes (14.3\%); nine, chamaephytes $(14.3 \%) ; 37$, hemicryptophytes (58.7\%); three, cryptophytes (4.8\%); and five, therophytes (7.9\%). In the seasonal cerrado, we sampled 107 species (Table 2), of which 28 were phanerophytes (26.2\%); 19 , chamaephytes $(17.7 \%)$; 54, hemicryptophytes (50.5\%); three, cryptophytes (2.8\%); and three, therophytes $(2.8 \%)$. These life-form spectra were not significantly different $\left(\chi^{2}=4.351\right.$, df $\left.=4, \mathrm{P}=0.361\right)$. The overall IDDs of the hyperseasonal and seasonal cerrados were, respectively, $0.950\left(\alpha_{\mathrm{SP}}=5.682\right.$ and $\left.\alpha_{\mathrm{LF}}=0.759\right)$ and $0.966\left(\alpha_{\mathrm{SP}}=11.889\right.$ and $\left.\alpha_{\mathrm{LF}}=0.883\right)$, values not significantly different $(\mathrm{P}=0.363$; Figure 1$)$.

When calculated for each season of the year, the only significant differences were those between February and November $(\mathrm{P}=0.016)$ and between May and November $(\mathrm{P}=0.022)$ in the hyperseasonal cerrado (Table 3$)$. In the hyperseasonal cerrado, the waterlogging decreased the IDD (Figure 2), and the seasonal variation of $\alpha_{\mathrm{SP}}$ was higher than of $\alpha_{L F}$ (Figure 3).

\section{Discussion}

In the life-form spectra of both cerrados, the most represented class was the hemicryptophytes. Hemicryptophytes and phanerophytes are generally well represented in cerrado life-form spectra (Batalha and Martins, 2002). Phanerophytes, however, were poorly represented in the cerrados we sampled and this may be a consequence of their open physiognomies. Although Raunkiaer's system was not originally proposed for physiognomic comparisons, Raunkiaer (1934) himself advocated that the life-form spectrum of a given site reflects the vegetation physiognomy. Thus, since the importance of trees and shrubs increases from open to closed cerrado physiognomies, hemicryptophytes should indeed prevail and phanerophytes should be almost absent in grassland cerrados (Batalha and Martins, 2002).

Even if therophytes are well represented in Venezuelan and African savannas (Batalha and Martins, 2002), we found an underproportion of them in both cerrados. The occurrence of therophytes in plant com- 
Table 1. Species, number of individuals and life-forms found in the hyperseasonal cerrado (18 $18^{\prime} 07^{\prime \prime} \mathrm{S}$ and $\left.52^{\circ} 57^{\prime} 56^{\prime \prime} \mathrm{W}\right)$, Emas National Park, central Brazil. Feb. = February 2002 (mid rainy season), May 2002 (late rainy season), Aug. = August 2002 (dry season), Nov. = November 2002 (early rainy season), $\mathrm{Ph}=$ phanerophyte, $\mathrm{Ch}=$ chamaephyte, $\mathrm{H}=$ hemicryptophyte, $\mathrm{Cr}=$ cryptophyte and $\mathrm{Th}=$ therophytes.

\begin{tabular}{|c|c|c|c|c|c|c|}
\hline Family/Species & Feb. & May & Aug. & Nov. & Total & Lf \\
\hline \multicolumn{7}{|l|}{ AMARANTHACEAE } \\
\hline Froelichia procera (Seub.) Pedersen & - & - & - & 1 & 1 & $\mathrm{H}$ \\
\hline Pfaffia helichrysoides (Moq.) Kuntze & - & - & - & 1 & 1 & $\mathrm{H}$ \\
\hline \multicolumn{7}{|l|}{ ANNONACEAE } \\
\hline Annona crassiflora Mart. & - & - & - & 1 & 1 & $\mathrm{Ph}$ \\
\hline \multicolumn{7}{|l|}{ ARECACEAE } \\
\hline Allagoptera campestris (Mart.) Kuntze & 3 & 6 & 3 & 3 & 15 & $\mathrm{Cr}$ \\
\hline Syagrus flexuosa (Mart.) Becc. & - & 6 & 4 & 17 & 27 & $\mathrm{Cr}$ \\
\hline \multicolumn{7}{|l|}{ ASTERACEAE } \\
\hline Asteraceae sp. 1 & - & - & 13 & - & 13 & Th \\
\hline Chromolaena squalida (DC.) King and H. Rob. & - & 5 & - & 2 & 7 & $\mathrm{Ch}$ \\
\hline Emilia coccinea (Sims.) Sweet & - & 1 & - & - & 1 & Th \\
\hline Erechtites hieracifolia (L.) Raf. ex DC. & 2 & 1 & - & - & 3 & $\mathrm{Th}$ \\
\hline Eupatorium campestre A. DC. & - & - & - & 1 & 1 & $\mathrm{H}$ \\
\hline \multicolumn{7}{|l|}{ CELASTRACEAE } \\
\hline Tontelea micrantha (Mart.) A. C. Smith & 5 & - & - & - & 5 & $\mathrm{Ph}$ \\
\hline \multicolumn{7}{|l|}{ CONVOLVULACEAE } \\
\hline Ipomoea procurrens Meissn. & - & - & - & 1 & 1 & $\mathrm{Ph}$ \\
\hline \multicolumn{7}{|l|}{ EBENACEAE } \\
\hline Diospyros hispida A. DC. & - & - & 2 & - & 2 & $\mathrm{Ph}$ \\
\hline \multicolumn{7}{|l|}{ ERYTHROXYLACEAE } \\
\hline Erythroxylum campestre A. St-Hill. & - & - & - & 8 & 8 & $\mathrm{Ph}$ \\
\hline \multicolumn{7}{|l|}{ EUPHORBIACEAE } \\
\hline Croton pohlianus Müll. Arg. & - & - & - & 1 & 1 & $\mathrm{H}$ \\
\hline \multicolumn{7}{|l|}{ FABACEAE } \\
\hline Acosmium subelegans (Mohl.) Yakovlev & 11 & 28 & 7 & 14 & 60 & $\mathrm{Ph}$ \\
\hline Andira laurifolia Benth. & 16 & 41 & - & - & 57 & $\mathrm{Ch}$ \\
\hline Galactia dimorpha Burk. & - & 13 & - & - & 13 & $\mathrm{H}$ \\
\hline Galactia martii A. DC. & 6 & 9 & 4 & 18 & 37 & $\mathrm{H}$ \\
\hline Mimosa gracilis Benth. & - & - & - & 3 & 3 & $\mathrm{Ch}$ \\
\hline \multicolumn{7}{|l|}{ FLAUCORTIACEAE } \\
\hline Casearia sp. & - & - & - & 1 & 1 & $\mathrm{H}$ \\
\hline \multicolumn{7}{|l|}{ IRIDACEAE } \\
\hline Sisyrinchium vaginatum Spreng. & 1 & 1 & - & 3 & 5 & $\mathrm{Cr}$ \\
\hline \multicolumn{7}{|l|}{ LAMIACEAE } \\
\hline Eriope crassipes Benth. & - & 1 & - & - & 1 & $\mathrm{H}$ \\
\hline Hyptis adpressa A. St-Hill. ex Benth. & - & 28 & - & 15 & 43 & $\mathrm{H}$ \\
\hline Oсітит sp. & 4 & - & - & 125 & 129 & $\mathrm{H}$ \\
\hline \multicolumn{7}{|l|}{ LYTHRACEAE } \\
\hline Cuphea carthagenensis (Jacq.) Macbr. & - & - & - & 3 & 3 & $\mathrm{H}$ \\
\hline Cuphea sp. & - & 2 & - & - & 2 & $\mathrm{H}$ \\
\hline \multicolumn{7}{|l|}{ MALVACEAE } \\
\hline Byttneria oblongata Pohl. & 4 & 5 & 2 & 3 & 14 & $\mathrm{H}$ \\
\hline Peltaea edouardii (Hochr.) Krapov. and Cristóbal & - & - & - & 1 & 1 & $\mathrm{H}$ \\
\hline \multicolumn{7}{|l|}{ MYRTACEAE } \\
\hline Eugenia angustissima O. Berg & - & 9 & 4 & 46 & 59 & $\mathrm{H}$ \\
\hline
\end{tabular}


Table 1. Continued...

\begin{tabular}{|c|c|c|c|c|c|c|}
\hline Family/Species & Feb. & May & Aug. & Nov. & Total & Lf \\
\hline Eugenia calycina Cambess. & - & 33 & 48 & - & 81 & $\mathrm{Ch}$ \\
\hline Eugenia complicata $\mathrm{O}$. Berg & 50 & - & - & 17 & 67 & $\mathrm{Ch}$ \\
\hline Eugenia cristaensis $\mathrm{O}$. Berg & - & 3 & - & - & 3 & $\mathrm{H}$ \\
\hline Eugenia livida $\mathrm{O}$. Berg & 1 & - & - & - & 1 & $\mathrm{Ph}$ \\
\hline Myrcia rhodeosepala Kiaersk. & 2 & 1 & 5 & - & 8 & $\mathrm{Ph}$ \\
\hline Myrcia torta A. DC. & - & - & - & 7 & 7 & $\mathrm{Ch}$ \\
\hline Myrcia uberavensis O. Berg & 1 & - & - & - & 1 & $\mathrm{Ph}$ \\
\hline Myrciaria delicatula (DC.) O. Berg. & - & 69 & 1 & 23 & 93 & $\mathrm{H}$ \\
\hline Myrtaceae sp. 1 & - & - & 2 & - & 2 & $\mathrm{Ch}$ \\
\hline Psidium australe Cambess. & 23 & - & 6 & 7 & 36 & $\mathrm{Ch}$ \\
\hline \multicolumn{7}{|l|}{ POACEAE } \\
\hline Andropogon bicornis $\mathrm{L}$. & - & 8 & - & - & 8 & $\mathrm{H}$ \\
\hline Andropogon leucostachyus (Hack.) Hack. & 122 & 421 & 477 & 199 & 1219 & $\mathrm{H}$ \\
\hline Axonopus derbyanus Black & - & 4 & - & 5 & 9 & $\mathrm{H}$ \\
\hline Brachiaria decumbens Stapf & 5 & 1 & 2 & - & 8 & $\mathrm{H}$ \\
\hline Eragrostis articulata (Schrank) Nees & - & 1 & - & 1 & 2 & Th \\
\hline Gymnopogon foliosus (Willd.) Nees & - & - & 7 & 2 & 9 & $\mathrm{H}$ \\
\hline Hyparrhenia rufa (Nees) Stapf. & - & 1 & - & - & 1 & $\mathrm{H}$ \\
\hline Ichnanthus procurrens (Nees ex Trin.) Sw. & - & 1 & 1 & - & 2 & $\mathrm{H}$ \\
\hline Loudetiopsis chrysothryx (Nees) Conert. & - & 17 & 47 & 8 & 72 & $\mathrm{H}$ \\
\hline Melinis minutiflora P. Beauv. & - & 7 & 2 & 30 & 39 & $\mathrm{H}$ \\
\hline Panicum parvifolium Lam. & 9 & 15 & 7 & - & 31 & $\mathrm{H}$ \\
\hline Panicum rudgei Roem. and Schult. & 7 & 28 & 20 & 2 & 57 & $\mathrm{H}$ \\
\hline Paspalum gemniiniflorum Steud. & - & 1 & - & - & 1 & $\mathrm{H}$ \\
\hline Paspalum pectinatum Nees & - & 4 & - & 4 & 8 & $\mathrm{H}$ \\
\hline Poaceae sp. 1 & - & - & - & 181 & 181 & $\mathrm{H}$ \\
\hline Poaceae sp. 2 & - & - & 1 & - & 1 & $\mathrm{H}$ \\
\hline Poaceae sp. 3 & - & - & 5 & - & 5 & $\mathrm{H}$ \\
\hline Poaceae sp. 4 & - & - & 1 & - & 1 & $\mathrm{H}$ \\
\hline Rynchelytrum repens (Nees) C.E.Hubb. & - & - & - & 1 & 1 & Th \\
\hline Schizachyrium condensatum (Kunth) Nees & - & - & 5 & - & 5 & $\mathrm{H}$ \\
\hline Tristachya leiostachya Nees & - & - & - & 78 & 78 & $\mathrm{H}$ \\
\hline \multicolumn{7}{|l|}{ SCROPHULARIACEAE } \\
\hline Scoparia dulcis L. & - & - & 1 & - & 1 & $\mathrm{Ch}$ \\
\hline \multicolumn{7}{|l|}{ UNKNOWN } \\
\hline Unknown & - & - & - & 1 & 1 & $\mathrm{H}$ \\
\hline Total & 272 & 771 & 677 & 834 & 2554 & - \\
\hline
\end{tabular}

munities is related to the harshness of growing conditions (Bazzaz and Morse, 1991; Cornelissen et al., 2003). Unfavorable growing conditions for part of the year, however, do not prevent perennials from appearing, since they favor selection of other strategies, such as dormant structures that enable hemicryptophytes to survive conditions detrimental to herbaceous growth (Bazzaz and Morse, 1991). Therefore, even the environmental constraints resulting from drought and waterlogging in the hyperseasonal cerrado should not be extreme enough to favor the strategy of therophytes.
The analysis of life-form spectra indicated that there should be a high functional redundancy among cerrado species. Life-form synthesizes various morphological traits related to responses of plants to climate, soil resource, competitive strength, and disturbances (Schulze, 1982; Cornelissen et al., 2003). Nevertheless, the life-form spectrum of the hyperseasonal cerrado, when compared to those of the seasonal cerrado, was not altered by waterlogging, even though the number of species decreased almost by half. Where the environment situations are spatiotemporally heterogeneous, as in savannas, species are adapted 
Table 2. Species, number of individuals and life-forms found in the seasonal cerrado $\left(18^{\circ} 17^{\prime} 34^{\prime \prime} \mathrm{S}\right.$ and $52^{\circ} 58^{\prime} 12^{\prime}$ ' W), Emas National Park, central Brazil. Feb. = February 2002 (mid rainy season), May 2002 (late rainy season), Aug. = August 2002 (dry season), Nov. = November 2002 (early rainy season), $\mathrm{Ph}=$ phanerophyte, $\mathrm{Ch}=$ chamaephyte, $\mathrm{H}=$ hemicryptophyte, $\mathrm{Cr}=$ cryptophyte and $\mathrm{Th}=$ therophytes.

\begin{tabular}{|c|c|c|c|c|c|c|}
\hline Family/Species & Feb. & May & Aug. & Nov. & Total & Lf \\
\hline \multicolumn{7}{|l|}{ AMARANTHACEAE } \\
\hline Froelichia procera (Seub.) Pedersen & 9 & 1 & 1 & 1 & 12 & $\mathrm{H}$ \\
\hline Pfaffia helichrysoides (Moq.) Kuntze & - & - & - & 4 & 4 & $\mathrm{H}$ \\
\hline \multicolumn{7}{|l|}{ ANACARDIACEAE } \\
\hline Anacardium humile A. St-Hil. & - & 2 & - & 4 & 6 & $\mathrm{Ch}$ \\
\hline \multicolumn{7}{|l|}{ ANNONACEAE } \\
\hline Annona crassiflora Mart. & 9 & 4 & 1 & 2 & 16 & $\mathrm{Ph}$ \\
\hline \multicolumn{7}{|l|}{ ANNONACEAE } \\
\hline Annona warmingiana Mello-Silva and Pirani & 4 & - & - & 1 & 5 & $\mathrm{H}$ \\
\hline \multicolumn{7}{|l|}{ ARECACEAE } \\
\hline Allagoptera campestris (Mart.) Kuntze & 5 & 6 & 1 & 2 & 14 & $\mathrm{Cr}$ \\
\hline Syagrus flexuosa (Mart.) Becc. & 12 & 9 & 9 & 7 & 37 & $\mathrm{Cr}$ \\
\hline \multicolumn{7}{|l|}{ ASTERACEAE } \\
\hline Aspilia leucoglossa Malme & 1 & - & 3 & 1 & 5 & $\mathrm{H}$ \\
\hline Asteraceae sp. 2 & - & 11 & - & - & 11 & $\mathrm{H}$ \\
\hline Calea clausseniana Baker & - & - & - & 2 & 2 & $\mathrm{H}$ \\
\hline Calea cuneifolia A. DC. & - & - & - & 5 & 5 & $\mathrm{H}$ \\
\hline Elephantopus biflorus Less. & - & - & 1 & - & 1 & $\mathrm{H}$ \\
\hline Vernonia herbacea (Vell.) Rusby & - & - & - & 2 & 2 & $\mathrm{H}$ \\
\hline Wedelia macedoi $\mathrm{H}$. Rob. & 2 & - & - & - & 2 & $\mathrm{H}$ \\
\hline \multicolumn{7}{|l|}{ BIGNONIACEAE } \\
\hline Tabebuia ochracea (Cham.) Standl. & 5 & 3 & - & - & 8 & $\mathrm{Ph}$ \\
\hline \multicolumn{7}{|l|}{ BURSERACEAE } \\
\hline Protium ovatum Engl. & - & - & 1 & 1 & 2 & $\mathrm{Ph}$ \\
\hline \multicolumn{7}{|l|}{ CELASTRACEAE } \\
\hline Tontelea micrantha (Mart.) A. C. Smith & 1 & - & 2 & 18 & 21 & $\mathrm{Ph}$ \\
\hline \multicolumn{7}{|l|}{ CONNARACEAE } \\
\hline Rourea induta Planch. & 1 & 1 & - & 3 & 5 & $\mathrm{Ph}$ \\
\hline \multicolumn{7}{|l|}{ CYPERACEAE } \\
\hline Bulbostylis junciformis Kuntze & - & 1 & 3 & - & 4 & $\mathrm{H}$ \\
\hline \multicolumn{7}{|l|}{ DILLENEACEAE } \\
\hline Davilla elliptica A. St-Hil. & 1 & 10 & - & - & 11 & $\mathrm{Ph}$ \\
\hline \multicolumn{7}{|l|}{ EBENACEAE } \\
\hline Diospyros hispida A. DC. & 9 & 5 & 13 & 5 & 32 & $\mathrm{Ph}$ \\
\hline \multicolumn{7}{|l|}{ ERYTHROXYLACEAE } \\
\hline Erythroxylum campestre A. St-Hill. & - & 11 & 8 & - & 19 & $\mathrm{Ph}$ \\
\hline Erythroxylum deciduum A. St-Hil. & 6 & - & - & - & 6 & $\mathrm{Ph}$ \\
\hline Erythroxylum suberosum A. St-Hil. & 5 & - & - & 3 & 8 & $\mathrm{Ph}$ \\
\hline \multicolumn{7}{|l|}{ EUPHORBIACEAE } \\
\hline Croton antisyphiliticus Mart. & 16 & 7 & 1 & - & 24 & $\mathrm{H}$ \\
\hline Croton lundianus Müll. Arg. & - & - & 2 & - & 2 & $\mathrm{H}$ \\
\hline Croton pohlianus Müll. Arg. & - & 1 & - & - & 1 & $\mathrm{H}$ \\
\hline Sebastiania bidentata (Mart.) Pax & - & - & 1 & - & 1 & $\mathrm{H}$ \\
\hline \multicolumn{7}{|l|}{ FABACEAE } \\
\hline Acosmium subelegans (Mohl.) Yakovlev & 4 & 7 & 4 & 13 & 28 & $\mathrm{Ph}$ \\
\hline Camptosema ellipticum (Desv.) Burkart & 2 & - & - & - & 2 & $\mathrm{Ph}$ \\
\hline
\end{tabular}


Table 2. Continued...

\begin{tabular}{|c|c|c|c|c|c|c|}
\hline Family/Species & Feb. & May & Aug. & Nov. & Total & Lf \\
\hline Chamaecrista nictitans (L.) Moench. & - & 1 & - & 1 & 2 & $\mathrm{Ch}$ \\
\hline Eriosema crinitum (Kunth) Gardner & 2 & - & - & 1 & 3 & $\mathrm{H}$ \\
\hline Eriosema longifolium Benth. & 3 & - & - & - & 3 & $\mathrm{H}$ \\
\hline Galactia decumbens (Benth.) Chodat and Hassl. & 4 & 1 & - & 4 & 9 & $\mathrm{H}$ \\
\hline Galactia dimorpha Burk. & - & - & - & 2 & 2 & $\mathrm{H}$ \\
\hline Galactia martii A. DC. & 2 & 10 & 11 & 5 & 28 & $\mathrm{H}$ \\
\hline Mimosa gracilis Benth. & 3 & 5 & - & - & 8 & $\mathrm{Ch}$ \\
\hline Mimosa polycephala Benth. & - & 3 & - & 3 & 6 & $\mathrm{Ch}$ \\
\hline Periandra mediterranea (Vell.) Taub. & - & 24 & - & - & 24 & $\mathrm{Ch}$ \\
\hline \multicolumn{7}{|l|}{ FLAUCORTIACEAE } \\
\hline Casearia sp. & 3 & - & - & - & 3 & $\mathrm{H}$ \\
\hline \multicolumn{7}{|l|}{ IRIDACEAE } \\
\hline Sisyrinchium vaginatum Spreng. & - & 1 & 3 & 2 & 6 & $\mathrm{Cr}$ \\
\hline \multicolumn{7}{|l|}{ LAMIACEAE } \\
\hline Eriope crassipes Benth. & - & 4 & 3 & - & 7 & $\mathrm{H}$ \\
\hline Hyptis adpressa A. St-Hill. ex Benth. & 2 & 9 & 2 & - & 13 & $\mathrm{H}$ \\
\hline Hyptis villosa Pohl ex Benth. & 3 & 1 & 1 & 16 & 21 & $\mathrm{H}$ \\
\hline Hyptis virgata Benth. & - & - & 1 & - & 1 & $\mathrm{H}$ \\
\hline Ocimum sp. & 34 & - & - & 28 & 62 & $\mathrm{H}$ \\
\hline \multicolumn{7}{|l|}{ LYTHRACEAE } \\
\hline Cuphea carthagenensis (Jacq.) Macbr. & 1 & 8 & 4 & 5 & 18 & $\mathrm{H}$ \\
\hline \multicolumn{7}{|l|}{ MALPIGHIACEAE } \\
\hline Byrsonima crassa Nied. & - & - & 2 & - & 2 & $\mathrm{Ph}$ \\
\hline Byrsonima guilleminiana A. Juss. & 3 & 3 & - & - & 6 & $\mathrm{Ch}$ \\
\hline \multicolumn{7}{|l|}{ MALVACEAE } \\
\hline Byttneria oblongata Pohl & 12 & 6 & - & 4 & 22 & $\mathrm{H}$ \\
\hline Peltaea edouardii (Hochr.) Krapov. and Cristóbal & 4 & 1 & - & 1 & 6 & $\mathrm{H}$ \\
\hline Waltheria douradinha A. St-Hil. & 7 & - & - & - & 7 & $\mathrm{H}$ \\
\hline \multicolumn{7}{|l|}{ MELASTOMATACEAE } \\
\hline Miconia ligustroides (A. DC.) Naud. & - & - & - & 10 & 10 & $\mathrm{Ph}$ \\
\hline \multicolumn{7}{|l|}{ MENISPERMACEAE } \\
\hline Cissampelos ovalifolia Ruiz and Pav. & - & - & 1 & - & 1 & $\mathrm{H}$ \\
\hline \multicolumn{7}{|l|}{ MYRTACEAE } \\
\hline Campomanesia pubescens (A. DC.) O. Berg & 80 & 72 & 9 & 31 & 192 & $\mathrm{Ph}$ \\
\hline Eugenia angustissima $\mathrm{O}$. Berg & 44 & 87 & 58 & 67 & 256 & $\mathrm{H}$ \\
\hline Eugenia aurata $\mathrm{O}$. Berg & - & 2 & - & 4 & 6 & $\mathrm{Ph}$ \\
\hline Eugenia bimarginata A. DC. & - & - & 1 & - & 1 & $\mathrm{Ph}$ \\
\hline Eugenia calycina Cambess. & 7 & 2 & 5 & - & 14 & $\mathrm{Ch}$ \\
\hline Eugenia complicata $\mathrm{O}$. Berg & - & 1 & - & 7 & 8 & $\mathrm{Ch}$ \\
\hline Eugenia piauhiensis $\mathrm{O}$. Berg & - & - & 5 & 2 & 7 & $\mathrm{Ph}$ \\
\hline Eugenia punicifolia (Kunth) A. DC. & - & 3 & - & 1 & 4 & $\mathrm{Ph}$ \\
\hline Myrcia guianensis A. DC. & - & 49 & 21 & - & 70 & $\mathrm{Ph}$ \\
\hline Myrcia rhodeosepala Kiaersk. & 11 & - & 8 & 11 & 30 & $\mathrm{Ph}$ \\
\hline Myrcia torta A. DC. & - & - & - & 1 & 1 & $\mathrm{Ch}$ \\
\hline Myrcia uberavensis O. Berg & 1 & 8 & - & - & 9 & $\mathrm{Ph}$ \\
\hline Myrciaria delicatula (DC.) Berg. & - & 91 & 246 & 233 & 570 & $\mathrm{H}$ \\
\hline Myrtaceae sp. 2 & 2 & - & - & - & 2 & $\mathrm{Ch}$ \\
\hline Myrtaceae sp. 3 & 19 & - & - & - & 19 & $\mathrm{Ch}$ \\
\hline
\end{tabular}


Table 2. Continued...

\begin{tabular}{|c|c|c|c|c|c|c|}
\hline $\begin{array}{r}\text { Family/Species } \\
\end{array}$ & Feb. & May & Aug. & Nov. & Total & Lf \\
\hline Psidium australe Camb. & 2 & 17 & 15 & 15 & 49 & $\mathrm{Ch}$ \\
\hline Psidium cinereum Mart. & 3 & 6 & - & - & 9 & $\mathrm{Ch}$ \\
\hline Psidium firmum $\mathrm{O}$. Berg & - & 3 & 2 & 13 & 18 & $\mathrm{Ch}$ \\
\hline Psidium laruotteanum Cambess. & 17 & 4 & 1 & 8 & 30 & $\mathrm{Ph}$ \\
\hline Psidium multiflorum Cambess. & - & - & 1 & - & 1 & $\mathrm{Ch}$ \\
\hline Psidium rufum Mart. ex A. DC. & 8 & - & - & 1 & 9 & $\mathrm{Ph}$ \\
\hline \multicolumn{7}{|l|}{ OCHNACEAE } \\
\hline Ouratea floribunda (A. St-Hil.) Engl. & - & 2 & 11 & - & 13 & $\mathrm{Ch}$ \\
\hline Ouratea nana (A. St-Hil.) Engl. & 4 & 4 & 1 & 14 & 23 & $\mathrm{Ch}$ \\
\hline Ouratea spectabilis (Mart.) Engl. & 13 & - & - & 1 & 14 & $\mathrm{Ph}$ \\
\hline \multicolumn{7}{|l|}{ POACEAE } \\
\hline Andropogon leucostachyus (Hack.) Hack. & - & 1 & - & 2 & 3 & $\mathrm{H}$ \\
\hline Anthaenantiopsis perforata (Nees) Parodi & 2 & - & 17 & - & 19 & $\mathrm{H}$ \\
\hline Aristida riparia Trin. & 2 & - & - & - & 2 & $\mathrm{H}$ \\
\hline Axonopus derbyanus Black & 4 & 8 & 57 & 23 & 92 & $\mathrm{H}$ \\
\hline Elionurus latiflorus Nees & 69 & 8 & - & 28 & 105 & $\mathrm{H}$ \\
\hline Elionurus muticus Kuntze & - & - & 1 & - & 1 & $\mathrm{H}$ \\
\hline Eragrostis articulata (Schrank) Nees & 5 & - & - & - & 5 & $\mathrm{Th}$ \\
\hline Gymnopogon foliosus (Willd.) Nees & - & 6 & 1 & 7 & 14 & $\mathrm{H}$ \\
\hline Leptosaccharum filiforme A. Camus & - & 22 & - & - & 22 & $\mathrm{H}$ \\
\hline Loudetiopsis chrysothryx (Nees) Conert. & - & 5 & 32 & 1 & 38 & $\mathrm{H}$ \\
\hline Panicum olyroides Kunth & - & - & 1 & - & 1 & $\mathrm{H}$ \\
\hline Panicum rudgei Roem. and Schult. & 2 & - & 23 & 1 & 26 & $\mathrm{H}$ \\
\hline Paspalum carinatum Humb. and Bonpl. ex Fleug. & - & 1 & - & 18 & 19 & $\mathrm{H}$ \\
\hline Paspalum gardnerianum Nees & - & - & 2 & - & 2 & $\mathrm{H}$ \\
\hline Paspalum lineare Trin. & - & 8 & - & - & 8 & $\mathrm{H}$ \\
\hline Paspalum pectinatum Nees & 7 & 1 & 1 & 2 & 11 & $\mathrm{H}$ \\
\hline Poaceae sp. 5 & - & 10 & - & 6 & 16 & $\mathrm{H}$ \\
\hline Poaceae sp. 6 & - & 4 & - & - & 4 & $\mathrm{H}$ \\
\hline Poaceae sp. 7 & - & - & 1 & - & 1 & $\mathrm{H}$ \\
\hline Rynchelytrum repens (Nees) C. E. Hubb. & 3 & 11 & - & - & 14 & Th \\
\hline Trachypogon sp. & - & - & 11 & 2 & 13 & $\mathrm{H}$ \\
\hline Tristachya leiostachya Nees & 88 & 277 & 160 & 319 & 844 & $\mathrm{H}$ \\
\hline \multicolumn{7}{|l|}{ RUBIACEAE } \\
\hline Diodia schumanii Standl. & - & - & - & 1 & 1 & $\mathrm{Th}$ \\
\hline Palicourea coriacea (Cham.) K. Schum. & - & - & 1 & 4 & 5 & $\mathrm{Ch}$ \\
\hline Tocoyena formosa (Cham. and Schl.) K. Schum. & - & - & - & 1 & 1 & $\mathrm{Ph}$ \\
\hline \multicolumn{7}{|l|}{ SAPINDACEAE } \\
\hline Serjania cissoides Radlk. & 1 & - & - & - & -1 & $\mathrm{Ph}$ \\
\hline \multicolumn{7}{|l|}{ SAPOTACEAE } \\
\hline Pradosia brevipes (Pierre) Penn. & 6 & 4 & - & - & 10 & $\mathrm{H}$ \\
\hline \multicolumn{7}{|l|}{ SOLANACEAE } \\
\hline Solanum subumbellatum Vell. & - & - & - & 3 & 3 & $\mathrm{Ch}$ \\
\hline \multicolumn{7}{|l|}{ TURNERACEAE } \\
\hline Piriqueta emasensis Arbo & - & - & - & 4 & 4 & $\mathrm{H}$ \\
\hline Total & 575 & 873 & 771 & 987 & 3206 & - \\
\hline
\end{tabular}




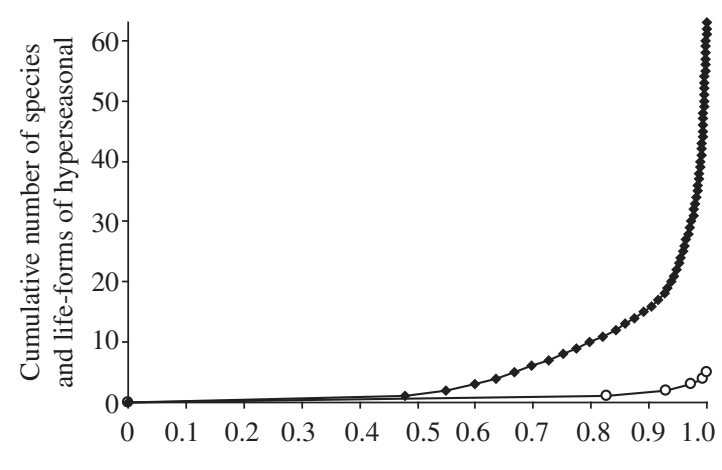

Cumulative relative abundances

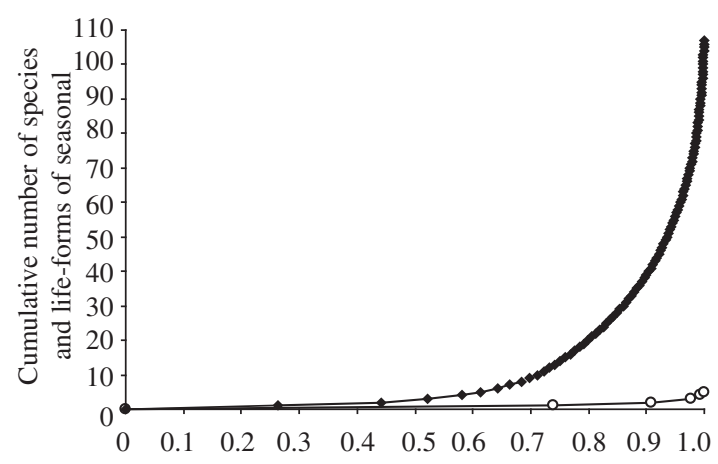

Cumulative relative abundances

$$
\text { —Species diversity ———Life-form diversity }
$$

Figure 1. Intrinsic diversity profile for species and life-form relative abundances of the hyperseasonal and seasonal cerrados in Emas National Park, central Brazil (approximately, $18^{\circ} 8^{\prime} 07^{\prime \prime} \mathrm{S}$ and $52^{\circ} 57^{\prime} 56^{\prime \prime} \mathrm{W}$ ).

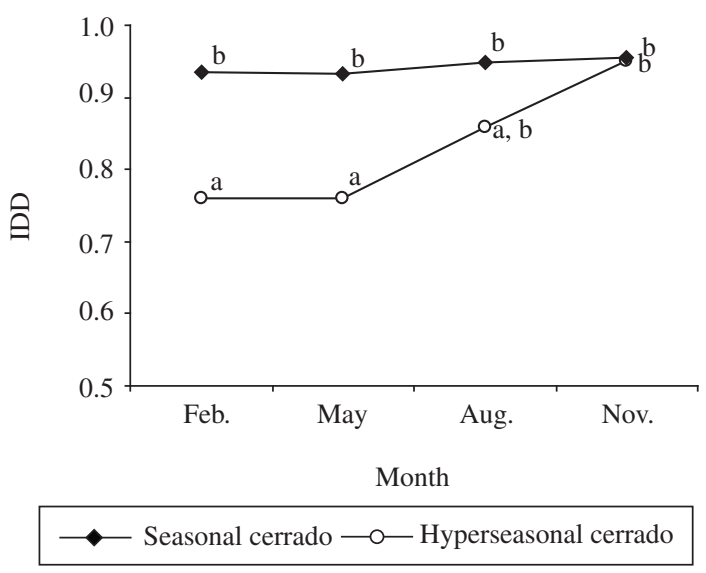

Figure 2. Variation of index of intrinsic diversity divergence (IDD) throughout the year (2003) in hyperseasonal and seasonal cerrados in Emas National Park, central Brazil (approximately, $18^{\circ} 17^{\prime} 34^{\prime \prime} \mathrm{S}$ and $52^{\circ} 58^{\prime} 12^{\prime \prime} \mathrm{W}$ ). Different letters indicate significantly different values $(\alpha=0.05)$. to dynamism and evolve strategies to respond to shifting opportunities (Fjeldsa and Lovett, 1997). Hence, the many responses of savanna species to environmental perturbations should explain the persistence of savanna communities within a broad range of environmental variation (Silva, 1996), from an excessive drought to a temporary waterlogging (Sarmiento, 1983).

The overall IDD comparison also pointed out that the cerrado species should be very functionally redundant. At the trait-group level of community organization, the environmental rules seem to determine the species convergence (Temperton et al., 2004; Fukami et al., 2005) against the neutral trait-group assembly driven by random sampling from regional species pools (Hubbell, 2001). Since the overall IDDs were not significantly different from a random resampling, we may conclude that the species convergences into life-forms in hyperseasonal and seasonal cerrados are, in general, similar. The high IDD values of the hyperseasonal and seasonal cerrados indicated that both communities are structurally very homogeneous. The dominance of only one life-form, hemicryptophytes, in the spectra of both cerrados justifies the high structural homogeneity of their grassland physiognomy.

On the other hand, the seasonal analyses corroborated that environmental constraints play the major role on community convergence into functional groups (Fukami et al., 2005). Comparing the IDDs throughout the year, we did not observe significant differences among the seasons in the seasonal cerrado. Although the water shortage in the dry season reduced the species diversity, drought did not affect species convergence in the seasonal cerrado, and the IDD values were constant throughout the year. On the contrary, we noticed a significant IDD variation throughout the year in the hyperseasonal cerrado. The IDD in November, in early rainy season, was higher than those in February, when the hyperseasonal cerrado was waterlogged, and in May, in late rainy season. When we considered the floras of both cerrados as a whole, we found no difference between the IDDs, but when we analyzed them throughout the year, we found seasonality only in the hyperseasonal cerrado. As a consequence, waterlogging shall modify cerrado species convergence into life-forms.

The seasonal comparisons also indicated that species abundance affects the convergence into functional groups, even though species composition variation does not (Fukami et al., 2005). The IDD measures the decrease from species to life-form diversity (Ricotta et al., 2002). Since life-form diversity was maintained throughout the year, structural changes in hyperseasonal cerrado were due to changes in species diversity. The IDDs of the hyperseasonal cerrado in February and May were lower, because the waterlogging reduced species diversity (Cianciaruso et al., 2005). Conversely, the IDD in November was higher, because the favorable moisture soil level of the early rainy season increased species diversity (Cianciaruso et al., 2005). Accordingly, annual variation of the hyperseasonal cerrado structure followed 
Table 3. Percentage of life-forms, species diversity $\left(\alpha_{\mathrm{SP}}\right)$, life-form diversity $\left(\alpha_{\mathrm{LF}}\right)$, and IDD in the hyperseasonal cerrado

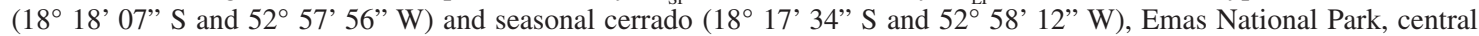
Brazil. $\mathrm{Ph}=$ phanerophyte, $\mathrm{Ch}=$ chamaephyte, $\mathrm{H}=$ hemicryptophyte, $\mathrm{Cr}=$ cryptophyte, and $\mathrm{Th}=$ therophytes. Different letters indicate significantly different values $(\alpha=0.05)$.

\begin{tabular}{lcrrrrrrrr}
\hline Vegetation form & Month & Ph & Ch & H & Cr & Th & $\alpha_{\text {SP }}$ & $\alpha_{\text {LF }}$ & IDD \\
\hline Hyperseasonal & Feb. & 7.4 & 32.7 & 58.1 & 1.1 & 0.7 & 2.794 & 1.037 & $0.761^{\mathrm{a}}$ \\
cerrado & May & 9.3 & 3.2 & 80.5 & 7.0 & 0.0 & 1.866 & 0.828 & $0.760^{\mathrm{a}}$ \\
& Aug. & 3.1 & 8.3 & 85.3 & 1.4 & 1.9 & 2.086 & 0.724 & $0.858^{\mathrm{a}, \mathrm{b}}$ \\
& Nov. & 4.3 & 4.3 & 88.1 & 3.1 & 0.2 & 4.327 & 0.692 & $0.950^{\mathrm{b}, \mathrm{c}}$ \\
Seasonal & Feb. & 30.4 & 7.5 & 57.7 & 3.0 & 1.4 & 9.789 & 1.098 & $0.936^{\mathrm{c}}$ \\
cerrado & May & 21.5 & 8.2 & 67.2 & 1.8 & 1.3 & 7.771 & 0.986 & $0.933^{\mathrm{c}}$ \\
& Aug. & 10.2 & 4.8 & 83.3 & 1.7 & 0.0 & 5.386 & 0.749 & $0.949^{\mathrm{c}}$ \\
& Nov. & 13.6 & 5.2 & 80.0 & 1.1 & 0.1 & 6.644 & 0.776 & $0.955^{\mathrm{c}}$ \\
\hline
\end{tabular}

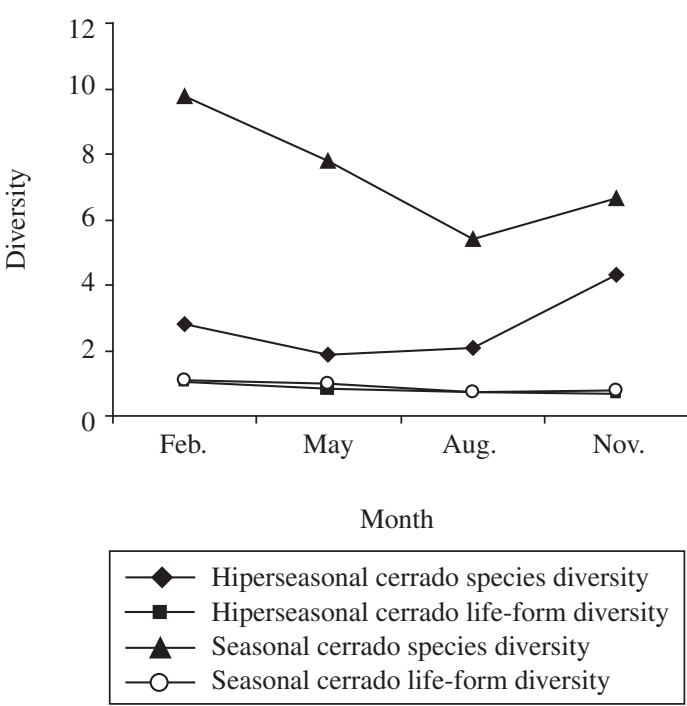

Figure 3. Variation of species $\left(\alpha_{\mathrm{SP}}\right)$ and life-forms diversity $\left(\alpha_{\mathrm{LF}}\right)$ throughout the year (2003) in hyperseasonal and seasonal cerrados in Emas National Park, central Brazil (approximately, $18^{\circ} 17^{\prime} 34^{\prime \prime} \mathrm{S}$ and $52^{\circ} 58^{\prime} 12^{\prime \prime} \mathrm{W}$ ).

the seasonality and its environmental constraints, being a consequence of the seasonal changes of species diversity rather than of life-form diversity.

Savannas should be more stable in functional than in floristic terms (Sarmiento, 1996). This was corroborated by the non-significant difference between the lifeform spectra and overall IDDs of the hyperseasonal and seasonal cerrados, as well as by the maintenance of lifeform diversity throughout the year. As redundant species within each life-form are not entirely equivalent, they have different tolerances to environmental constraints (Sarmiento, 1996). Consequently, changing conditions may result in a certain degree of floristic replacement, without major changes in functional groups. Thus, due to the high functional redundancy of cerrado plants, waterlogging in the hyperseasonal cerrado promotes seasonal changes in species convergence into life-forms by reducing species diversity.

Acknowledgements - We are grateful to FAPESP, for financial support; to CAPES, for the scholarship granted to the first author; to Ibama, for research permission; to the Emas National Park staff, for logistical assistance; to A.W. Valenti, for computational support on the randomization test; to São Paulo State Botanical Institute, UNB and IBGE herbaria staff; to P.K. Amorim, C.A. Casali, M.V. Cianciaruso, A.V.F. Jardim, H.F. Lima, R.A. Miotto, F. Oliveira, O.L. Silva, and M.W. Valenti, for help in field; and to the taxonomists T.S. Filgueiras, R.C. Oliveira, and M.A. Silva, for their assistance in the identification of species.

\section{References}

AMORIN, PK. and BATALHA, MA., 2006. Soil characteristics of a hyperseasonal cerrado compared to a seasonal cerrado and a floodplain grassland: implications for plant community structure. Braz. J. Biol.= Rev. Bras. Biol., vol. 66, no. 2b, p. 661-670.

BATALHA, MA., CIANCIARUSO, MV., SILVA, IA. and DELITTI, WCB., 2005. Hyperseasonal cerrado, a new Brazilian vegetation form. Braz. J. Biol.=Rev. Bras. Biol., vol. 65, no. 4, p. 735-738.

BATALHA, MA. and MARTINS, FR., 2002. Life-form spectra of Brazilian cerrado sites. Flora, vol. 197, no. 6, p. 452-460.

BAZZAZ, FA. and MORSE SR., 1991. Annual plants: potential responses to multiple stresses. In MOONEY, HA., WINNER, WE. and PELL, EJ. (eds). Response of plants to multiple stresses. London: Academic.

BEGON, M., HARPER, JL. and TOWNSEND, CR., 1996. Ecology: individuals, populations and communities. Oxford: Blackwell. 945 p.

CHASE, JM., 2003. Community assembly: when should history matter? Oecologia, vol. 136, no. 4, p. 489-498.

CIANCIARUSO, MV., BATALHA, MA. and SILVA, IA., 2005. Seasonal variation of a hyperseasonal cerrado in Emas National Park, central Brazil. Flora, vol. 200, no. 4, p. 345-353.

CORNELISSEN, JHC., LAVOREL, S., GARNIER, E., DÍAZ, S., BUCHMANN, N., GURVICH, DE., REICH, PB., STEEGE, H., MORGAN, HD., Van-Der-HEIJDEN, MGA., PAUSAS, 
JG. and POORTER, H., 2003. A handbook of protocols for standardised and easy measurement of plant functional traits worldwide. Aust. J. Bot., vol. 51, no. 4, p. 335-380.

CRAWFORD, RM., 1982. Physiological response to flooding. In LANGE, OL., NOBEL, PS., OSMOND, CB. and ZIEGLER, H. (eds.). Physiological plant ecology. II. Encyclopedia of plant physiology, vol. 12B, Berlin: Springer.

FJELDSA, J. and LOVETT, J., 1997. Biodiversity and environmental stability. Biodiversity Conserv., vol. 6, no. 3, p. 315-323.

FUKAMI, T., BEZEMER, TM., MORTIMER, SR. and Van-DerPUTTEN, WH., 2005. Species divergence and trait convergence in experimental plant community assembly. Ecol. Lett., vol. 8, no. 12 , p. 1283-1290.

GENTRY, AH., 1988. Changes in plant community diversity and floristic composition on environmental and geographic gradients. Ann. Mo. Bot. Gard., vol. 75, no. 1, p. 1-34.

HUBBELL, SP., 2001. The unified neutral theory of biodiversity and biogeography. Princeton: Princeton University. 375 p.

KINZIG, AP., LEVIN, SA., DUSHOFF, J. and PACALA, S., 1999. Limiting similarity, species packing, and system stability for hierarchical competition-colonization models. Am. Nat., vol. 153 , no. 4, p. 371-383.

KOSLOWSKI, TT., 1997. Responses of woody plants to flooding and salinity. Tree physiology monograph, no.1. Victoria: Heron Publishing. 29 p.

MANLY, BFJ., 1997. Randomization, bootstrap and Monte Carlo methods in biology. London: Chapman and Hall. 399 p.

MUELLER-DOMBOIS, D. and ELLENBERG, H., 1974. Aims and methods of vegetation ecology. New York: John Wiley and Sons. 547 p.

PATIL, GP. and TAILIE, C., 1979. An overview of diversity. In GRASSLE, F., SMITH, WK., PATIL, GP., and TAILLIE, C. (eds.). Ecological diversity in theory and practice. Fairland: International Cooperative.

PATIL, GP. and TAILIE, C., 1982, Diversity as a concept and its measurement. J. Am. Stat. Assoc., vol. 77, no. 379, p. 48-567.
RAMOS-NETO, MB. and PIVELLO, VR., 2000. Lightning fires in a Brazilian savanna National Park: rethinking management strategies. Environ. Manage., vol. 26, no. 6, p. 675-684.

RAUNKIAER, C., 1934. The life forms of plants and statistical geography. Oxford: Claredon. $632 \mathrm{p}$.

RICOTTA, C., AVENA, G. and CHIARUCCI, A., 2002. An index of divergence from species to life-form diversity based on the notion of intrinsic diversity ordering. Plant Ecol., vol. 165, no. 2 , p. $217-222$.

SARMIENTO, G., 1983. The savannas of tropical America. In GOODALL, DW (ed.). Ecosystems of the world - tropical savannas. Amsterdam: Elsevier.

-, 1996. Biodiversity and water relations in tropical savannas. In SOLBRIG, OT., MEDINA, E. and SILVA, JF. (eds.). Biodiversity and savanna ecosystem processes: a global perspective. Berlin: Springer.

SARMIENTO, G. and MONASTERIO, M., 1983. Life forms and phenology. In BOURLIÈRE, F. (ed.). Ecosystems of the world: tropical savannas. Amsterdam: Elsevier.

SARMIENTO, G., PINILLOS, M., SILVA, MP. and ACEVEDO, D., 2004. Effects of soil water regime and grazing on vegetation diversity and production in a hyperseasonal savanna at the Apure Llanos, Venezuela. J. Trop. Ecology, vol. 20, no. 2, p. 209-220.

SCHULZE, ED., 1982. Plant life forms and their carbon, water and nutrient relations. In LANGE, OL., NOBEL, PS., OSMOND, CB. and ZIEGLER, H. (eds.). Physiological plant ecology. II. Encyclopedia of plant physiology, vol. 12B. Berlin: Springer.

SILVA, JF., 1996. Biodiversity and stability in tropical savannas. In SOLBRIG, OT., MEDINA, E. and SILVA, JF. (eds.). Biodiversity and savanna ecosystem processes: a global perspective. Berlin: Springer.

TEMPERTON, VM., HOBBS, RJ., NUTTLE, T. and HALLE, S., 2004. Assembly rules and restoration ecology: bridging the gap between theory and practice. Washington: Island. $439 \mathrm{p}$.

WESTOBY, M., 1998. A leaf-height-seed (LHS) plant ecology strategy scheme. Plant Soil, vol. 199, no. 2, p. 213-227

ZAR, JH., 1999. Biostatistical analysis. New Jersey: Prentice Hall. 663 p. 